\title{
PAPER \\ Driving Voltage Analysis for Fast Response of Waveguide Optical Switch Based on Movement of Liquid Droplet Driven by Electrostatic Force
}

\author{
Takuji IKEMOTO $^{\dagger \mathrm{a})}$, Nonmember and Yasuo KOKUBUN ${ }^{\dagger b)}$, Fellow $^{2}$
}

\begin{abstract}
SUMMARY The electrostatic force required for the driving of liquid droplet injected in a microchannel was studied to obtain the guiding principle to reduce the driving voltage of waveguide optical switch based on the movement of droplet. We analytically calculated the relation between the threshold voltage and velocity of droplet and the surface roughness of microchannel, and clarified some unconfirmed parameters by comparing experimental results and aeromechanical analysis. The driving of droplet in a microchannel was best analyzed using the Hagen-Poiseuille flow theory, taking into account the movement of both ends of the droplet. When the droplet is driven by some external force, a threshold of the external force occurs in the starting of movement, and hysteresis occurs in the contact angle of the droplet to the side wall of the microchannel. The hysteresis of contact angle is caused by the roughness of side wall. In our experiment, the threshold voltage ranged from 200 to $350 \mathrm{~V}$ and the switching time from 34 to $36 \mathrm{~ms}$. The velocity of droplet was evaluated to be $0.3-0.4 \mathrm{~mm} / \mathrm{s}$ from these experimental results. On the other hand, the measured angle distribution of side wall roughness ranged from 30 to 110 degrees, and the threshold voltage was evaluated to be $100-320 \mathrm{~V}$, showing a good agreement with experimental results. The reduction of threshold voltage can be realized by smoothing the side wall roughness of microchannel. The switching time of $10 \mathrm{~ms}$, which is required for the optical stream switch, can be obtained by shortening the horizontal spot size down to $1.5 \mu \mathrm{m}$.

key words: optical switch, microelectro mechanical system, microchannel, droplet, electrostatic force
\end{abstract}

\section{Introduction}

In order to guarantee the reliability and flexibility of optical network system, optical switches are indispensable to the routing exchange. Most of conventional optical switches utilize the OEO signal conversion and the electric routing control. In this case, since the electric circuit cannot process and transmit the wavelength multiplexed signal, it must be demultiplexed, processed, and again multiplexed to be transmitted to their new route. In other words, when the number of multiplexed wavelength is increased with the data traffic, the electric processing circuit in the routing node must be expanded in proportion to the number of multiplexed wavelength.

As a result, the component size and the electric power consumption increase, and the signal processing capacity of the integrated circuit semiconductor reaches its limit.

\footnotetext{
Manuscript received November 30, 2007.

Manuscript revised May 31, 2008.

†The authors are with the Graduate School of Eng., Yokohama National Univ., Yokohama-shi, 240-8501 Japan.

a) E-mail: takuji@koklab.seg.ynu.ac.jp

b)E-mail: ykokubun@ynu.ac.jp

DOI: 10.1093/ietele/e91-c.12.1923
}

Therefore, in the WDM optical fiber network, the space division optical switch is necessary.

In this work, we aimed at developing a waveguide type space division optical switch with low power consumption for the optical stream switch system, which is promising to the near future signal routing system.

In the optical stream switch system, the fiber connection is switched among fixed nodes via an optical switch while the unconnected optical pass is invisible to the network. In this system, since the optical passes are not frequently and rapidly exchanged, the high-speed characteristic is not that much required for the optical switch. Therefore, the following properties are required for the optical switch:

1. Transmission loss: less than $-2 \mathrm{~dB}$,

2. Extinction ratio: $60 \mathrm{~dB}$ or better,

3. Scale of switch: several dozen $\times$ several dozen or more,

4. Reliability: longer than ten years,

5. Switching speed: faster than milli-second,

6. Polarization independent and mode independent,

Among these, the properties 1 to 3 are important. The space division optical switch is divided into the bulk type: one is single mode fibers and/or waveguides that are switched by an actuator [1] and the other integrated optic type. For the integrated optic type, the MEMS (Micro-ElectroMechanical Systems) type has advantages such as high precision of fabrication resulting from the IC production technology and high extinction ratio due to mechanical exchanging of optical path. Although the switching time is as slow as several milli-second, it is sufficient for the optical stream switch system.

There have been proposed and demonstrated some optical switches which were fabricated by the MEMS technology. Among them, two types are widely used; one is the mirror type where the optical pass being exchanged in the three dimensional space using micro-mirror [2] and the other the integrated waveguide type [3], [4] where the optical path being exchanged at the intersection of crossed optical waveguides.

In the mirror type optical switch, the direction of the optical beam emitted from the input fiber is controlled by changing the angle of the MEMS-type micro-mirror and is redirected and focused to the output fiber. In this type, however, a large-scale control circuit is necessary for sophisti- 
cated and precise control of the direction of micro-mirror [2], and there is a problem of the mirror drift which results in the lack of long-term reliability [5].

For the optical waveguide-type switch in which the optical path is exchanged at the intersection of crossed optical waveguides, a microchannel is formed so that it acts as a reflection mirror to the crossed waveguide, aided with matching oil inserted in the microchannel. The transmission or the reflection of the optical beam is switched via changes in refractive index caused by presence or absence of matching oil inside the microchannel. The matching oil is driven by thermal capillary phenomenon in the microchannel. In this type, however, since the matching oil is driven by heat, the electric power consumption is large and it is not suited for high density integration.

Although there are several problems in the space division optical switches using the MEMS technology, the optical waveguide type is suitable for the integration, and the control is not so sophisticated as the space mirror type.

In fact, in the waveguide type MEMS optical switch developed by the research group of NTT [3], they demonstrated the switching time of $6 \mathrm{~ms}$, electric power consumption of $0.15 \mathrm{~W}$, crosstalk of less than $60 \mathrm{~dB}$, and transmission losses of $0.11 \mathrm{~dB}$ at through port and $1.3 \mathrm{~dB}$ at reflection port. These characteristics are sufficient for optical stream switch.

For the reason, we aimed to demonstrate an waveguidetype space division optical switch in which the matching oil is driven by an electric field instead of thermal capillary phenomenon [6], [7]. In thiswork, we elucidated theoretically and experimentally the relationship between the response time and driving voltage of an optical switch, which drives matching oil in the microchannel with electric field.

\section{Structure and Operation Principle of Optical Switch}

Figure 1 shows the perspective view of the optical switch.

The crossed optical waveguide was fabricated on a $\mathrm{Si}$ substrate using $\mathrm{Ta}_{2} \mathrm{O}_{5}-\mathrm{SiO}_{2}(\mathrm{n}=1.560$ at $\lambda=1550 \mathrm{~nm})$ as the core and $\mathrm{SiO}_{2}(\mathrm{n}=1.455$ at $\lambda=1550 \mathrm{~nm})$ as the cladding material. At the intersection of the crossed waveguides, a microchannel is formed with the half angle of the crossing angle of crossed waveguide. Silicone oil with high chemical stability was injected into the microchannel as matching oil. In this work, KF56 (made by Shinetsu Silicone Co. Ltd., $\mathrm{n}_{D}=1.50$ at $\lambda=587.56 \mathrm{~nm}, \epsilon_{r}=2.8$ and viscosity $=15$ $\mathrm{Pa} \cdot \mathrm{s})$ was used. The upper electrode made of $\mathrm{Cr}$ was formed on the top of microchannel and connected to the electrode pad. The Si substrate was used as the lower electrode. In order to make Ohmic contact at the interface between the $\mathrm{Si}$ substrate and the metal electrode, Al was evaporated on the reverse side of the $\mathrm{Si}$ substrate. Circular liquid receivers were formed at both ends of microchannel so as to be open to the atmosphere. Matching oil was injected from these liquid receivers.

If there is no matching oil at the intersection part of crossed waveguide, the incident light is totally reflected at

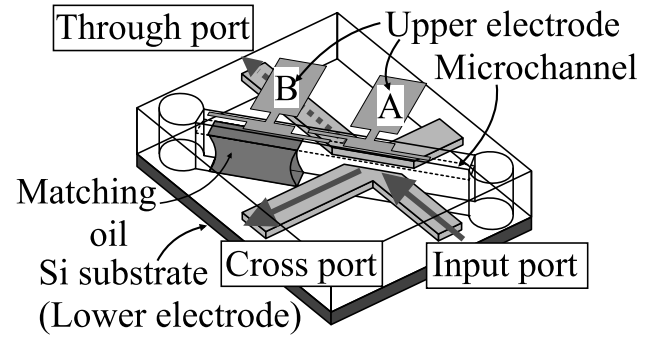

Fig. 1 Perspective view of optical waveguide switch based on microfluidics driven by electrostatic force.

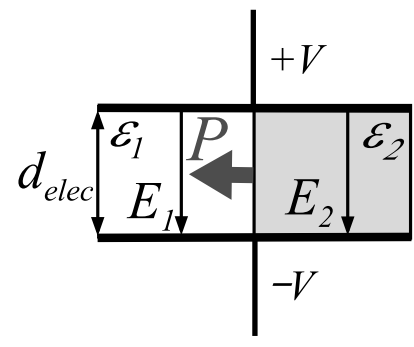

Fig. 2 Driving force of droplet (Maxwell's stress).

the sidewall of microchannel and is guided to the cross port. When the matching oil is moved to the intersection part of crossed waveguide, the incident light passes straight through to the through port.

The Maxwell's stress as shown in Fig. 2 was used as the driving force of the liquid droplet. The Maxwell's stress $\mathrm{P}[\mathrm{Pa}]$ is expressed as follow,

$$
\begin{aligned}
& E=\frac{V}{d_{\text {elec }}} \\
& P=\frac{1}{2}\left(\varepsilon_{2}-\varepsilon_{1}\right) E^{2},
\end{aligned}
$$

where $V[\mathrm{~V}]$ is the applied voltage, $d_{\text {elec }}[\mathrm{m}]$ is the distance of between upper and lower electrodes, $E[\mathrm{~V} / \mathrm{m}]$ is the electric field, and $\varepsilon_{1}$ and $\varepsilon_{2}[\mathrm{~F} / \mathrm{m}]$ are the permittivity of the dielectric materials of each layer, respectively.

In this study, $\varepsilon_{1}$ is 1.0 as the relative dielectric constant of air and $\varepsilon_{2}$ is 2.8 as the relative dielectric constant of matching oil. The Maxwell's stress $P$ is directed from low permittivity material to high permittivity material and is vertical to the dielectric boundary. In addition, it does not depend on the direction of electric field $E$.

Since this switch is driven by electric field, no energy is consumed in the steady state if there is no leak current. Since the driving of this optical switch is similar to charging a capacitor, switching energy is equivalent to that of the charge of a capacitor formed by upper and lower electrodes. The switching energy of capacitor type optical switch is much smaller than that of heater type optical switch [7].

In order to drive matching oil droplet, electric voltage is applied between the upper electrode on the target area to which the droplet should be moved and the lower electrode (Si substrate). When the matching oil is driven to the intersection part of the waveguide changing the state of switch 


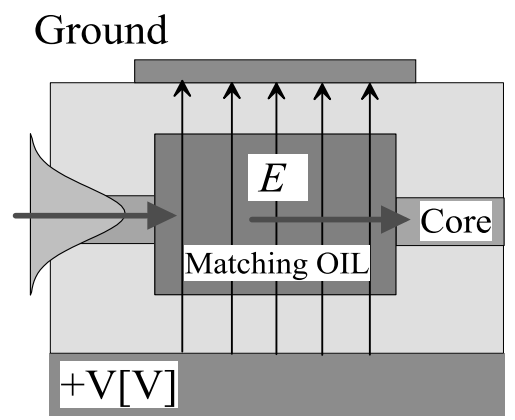

Fig. 3 Cross sectional view of crossing point of waveguide and microchannel.

from reflection to transmission state, the electric voltage is applied between lower electrodes and the upper electrode which is formed on the top surface of the waveguide intersection (upper electrode A in Fig. 1). On the other hand, when the matching oil is moved from the intersection part of the waveguide changing the state of switch from transmission to reflection state, the electric voltage is applied between lower electrodes and the upper electrode which is formed on the part other than the intersection part (upper electrode B in Fig. 1).

Figure 3 shows the cross sectional structure of microchannel. In order to form the upper electrode, the top surface of microchannel must be flat. In addition, since the Maxwell's stress is proportional to the square of electric field, the distance between the top and bottom electrodes should be as short as possible to reduce the driving voltage. In this work, we developed a new fabrication process of microchannel using the so-called damascene process, in which a sacrificial layer is formed in a groove and is flattened by polishing to form a flat cover layer [7].

\section{Theory of Droplet Flow}

The silicone oil can be regarded as the Newtonian fluid. In addition, the liquid flow in a microchannel is the laminar flow because the Reynolds' number Re is small.

Reynolds number Re is defined by

$$
\operatorname{Re}=\frac{\rho U_{0} D_{H}}{\mu}
$$

where $\mu[\mathrm{Pa} \cdot \mathrm{s}]$ is the viscosity of liquid, $U_{0}[\mathrm{~m} / \mathrm{s}]$ is the velocity of flux, and $D_{H}[\mathrm{~m}]$ is the hydraulic diameter of the tube [8].

Here, hydraulic diameter $D_{H}[\mathrm{~m}]$ is defined by

$$
D_{H}=\frac{2 w_{c h} t_{c h}}{w_{c h}+t_{c h}}
$$

for the rectangular tube with width $w_{c h}$ and height $t_{c h}$ [8].

For example, when water $\left(\mu=1 \times 10^{-3}[\mathrm{~Pa} \cdot \mathrm{s}]\right)$ is flowing in $=$ a square microchannel of $10 \mu \mathrm{m}$ side length with flux speed of $U_{0}=1 \mathrm{~mm} / \mathrm{s}$, the Reynolds' number is calculated to be $\operatorname{Re}=1 \times 10^{-2}$. This Reynolds' number is much

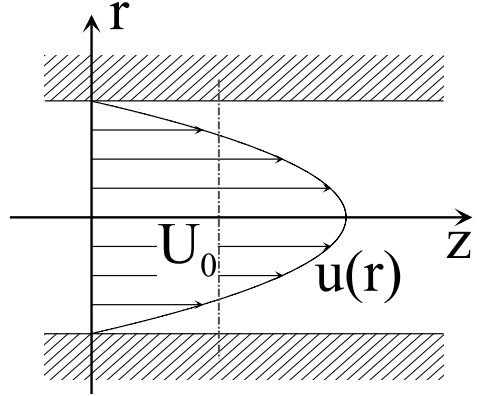

Fig. 4 Velocity profile of Hagen-Poiseuile flow.

smaller than the threshold number of 2300 giving the boundary between the laminar flow and the turbulent flow. Therefore, the continuous flow in a microchannel can be regarded as a Hagen-Poiseuille model which corresponds to the continuous flux of the Newtonian fluid with the laminar flow.

However, since the boundary exists on both ends of actual liquid droplet and it is not actually continuous flow, it is necessary to consider the dynamics of the boundary. In other words, the analytic model of the driving of liquid droplet in a microchannel should be treated as the Hagen-Poiseuille model taking into account the influence of the boundary.

\subsection{Continuous Flow Model}

Figure 4 shows a typical appearance of the Hagen-Poiseuille flow in the $\mathrm{z}$ direction. In the case of the Hagen-Poiseuille flow, the relationship between the pressure drop per unit length $d P / d z$ and the average velocity $U_{0}$ is given by

$$
\frac{d P_{\mathrm{H} . \mathrm{P}}}{d z}=\frac{32 \mu U_{0}}{D_{H}{ }^{2}}
$$

where $D_{H}$ is the hydraulic diameter of the microchannel which is given by Eq. (4) and $\mu$ is the viscosity of the fluid which is flowing in the microchannel [8].

The pressure drop $P$ over the liquid droplet length $L_{l q}$ can be evaluated by integrating Eq. (5) and is given by

$$
P_{\text {H.P. }}=\frac{32 \mu U_{0}}{D_{H}{ }^{2}} L_{l q}
$$

Assuming that the pressure drop $P$ given by Eq. (6) and the driving force given by Eq. (2) are balanced, the relation between the velocity of flux $U_{0}$ and the applied electric field $E$ is express by,

$$
U_{0}=\frac{D_{H}{ }^{2}\left(\varepsilon_{2}-\varepsilon_{1}\right)}{64 \mu L_{l q}} E^{2}
$$

It is seen from Eq. (7) that the velocity of the liquid $U_{0}$ is proportional to: the square of hydraulic diameter of the microchannel $D_{H}^{2}$; the square of electric field $E^{2}$ and the difference of permittivity $\left(\varepsilon_{2}-\varepsilon_{1}\right)$, and is inversely proportional to the viscosity of liquid and the length of the droplet.

The hydraulic diameter $D_{H}$ is given by Eq. (4) in terms 


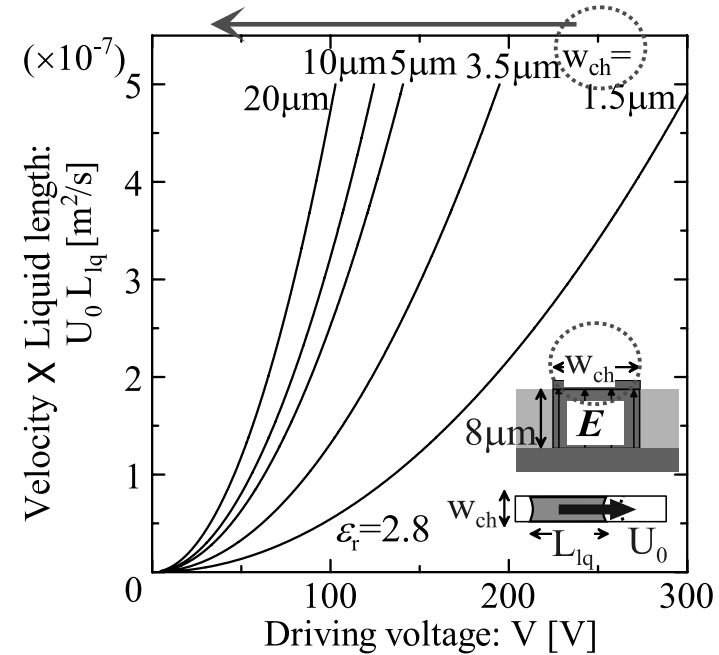

Fig.5 Calculated result of velocity vs. driving voltage for various width of microchannel (the height of microchannelis assumed to be $8 \mathrm{~mm}$ ).

of the width $w_{c h}$ and the height $t_{c h}$ of microchannel. Electrode distance $d_{\text {elec }}$ is determined by the height of microchannel $t_{c h}$. Figure 5 shows an analytical result of the relation between the applied voltage $V$ and the velocity of flux $U_{0}$ for the width of mcrochannel ranging from $1.5 \mu \mathrm{m}$ to $20 \mu \mathrm{m}$, assuming that the height of microchannel is equal to $8 \mu \mathrm{m}$ which is the same value as the device parameter in our experiment. Figure 6 shows the analytical result of the relation between the applied voltage $V$ and the velocity of flux $U_{0}$ for the height of microchannel $t_{c h}$ ranging from $1.5 \mu \mathrm{m}$ to $20 \mu \mathrm{m}$, assuming that the height of microchannel $t_{c h}$ is equal to the electrode distance $d_{\text {elec }}$ and the width of microchannel $w_{c h}$ is equal to $8 \mu \mathrm{m}$.

It is seen from Fig. 5 that the width of microchannel is wider and the driving voltage of liquid is lower. In addition, it is seen from Fig. 6 that when the height of microchannel is decreased, the driving voltage of liquid is decreased resulting from the fact that the field intensity becomes strong due to decrease of electrode distance although the liquid becomes hard to flow.

From the analytical results mentioned above, it is seen that in the continual fluid model the planiform microchannel with small aspect ratio is suited for low driving voltage. However in the planiform microchannel the diffraction loss of light beam increases [9]. In other words, the reduction of liquid driving voltage and the reduction of optical loss are in the relationship of trade-off with respect to the aspect ratio of microchannel. Therefore, the microchannel should be designed to decrease the optical loss so that the response time and the driving voltage satisfy their requirements.

\subsection{Dynamics at Surface Boundary}

The liquid used in our optical switch is not the continuous fluid but is a liquid droplet which has the boundary on both ends. Therefore, it is necessary to analyze the liquid driving property taking into account the influence of the boundary.

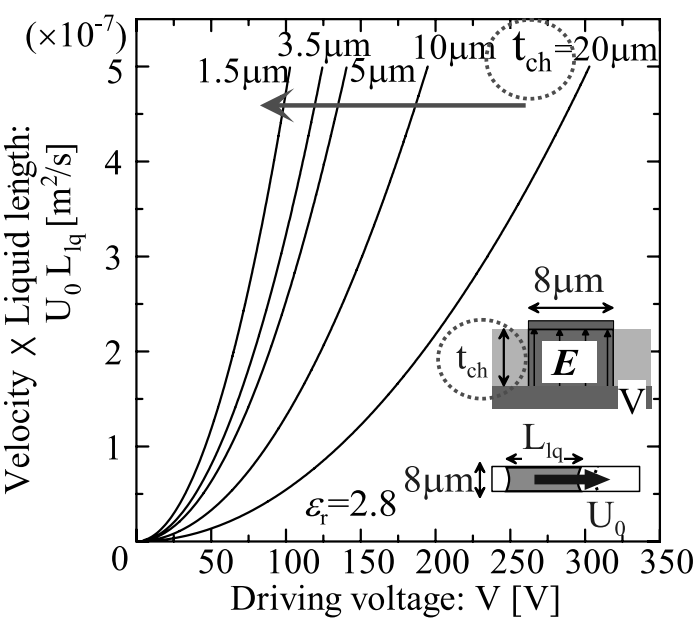

Fig. 6 Calculated result of velocity vs. driving voltage for various height of microchannle (the width of microchannel is assumed to be $8 \mathrm{~mm}$ ).

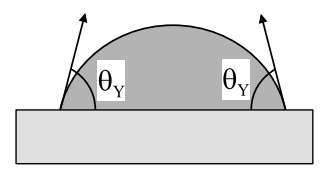

(a) Static contact angle

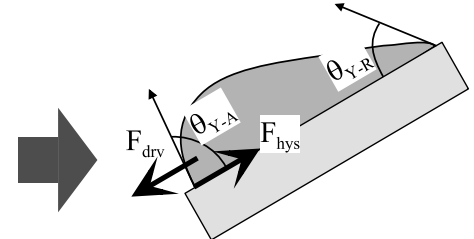

(b) Dynamic contact angle
Fig. 7 Definition of contact angles of droplet layed on a horizontal plate.

As shown in Fig. 7, the liquid droplet laid on a horizontal plate stands still with a certain stationary contact angle of $\theta_{Y}$. When the baseplate is tilted, the droplet starts to move at a certain tilt angle of plate because the driving force due to the gravity becomes large. In other words, the boundary between the liquid and the vapor starts to move when the front contact angle and the rear contact angle are equal to certain contact angles $\theta_{Y-A}$ and $\theta_{Y-R}$, respectively.

Here $\theta_{Y-A}>\theta_{Y-R}$ and the drag force $F_{\text {hys }}$ which oppose to the movement of the liquid droplet is expressed by

$$
F_{\text {hys }}=\gamma w_{l q}\left(\cos \theta_{Y-R}-\cos \theta_{R-A}\right)
$$

where $\gamma$ is the surface tension of the liquid, and $w_{l q}$ is the width of the liquid droplet [10].

This phenomenon in which $\theta_{Y-A}$ and $\theta_{Y-R}$ are different is called the contact angle hysteresis. One of the origins of contact angle hysteresis is the roughness of the baseplate surface. When the largest angle of roughness of the surface is designated as $\theta_{\sigma-\max }$, the relationship between the stationary contact angle $\theta_{Y}$ and the dynamic contact angles $\theta_{Y-A}$ and $\theta_{Y-R}$ is expressed by the following equations [11].

$$
\theta_{Y-A}=\theta_{Y}+\theta_{\sigma-\max }
$$

and

$$
\theta_{Y-R}=\theta_{Y}-\theta_{\sigma-\max } .
$$

In the optical switch shown in Fig. 8, when the liquid 


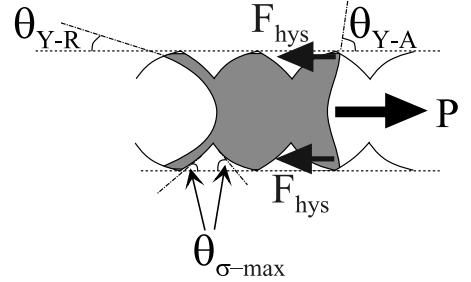

Fig. 8 Definition of contact angles of droplet injected in a microchannel.

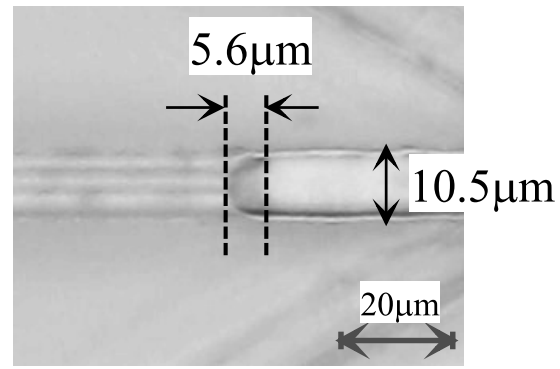

Fig. 9 Microscopic view of silicone oil meniscus injected in a microchannel.

droplet in the microchannel is driven by some external force, similar phenomena occurs. The liquid droplet does not start to move until the contact angles of front and rear sides are equal to certain contact angles $\theta_{Y-A}$ and $\theta_{Y-R}$, respectively.

Figure 9 shows the optical microscope photograph of the meniscus of silicone oil droplet (KF56, made by Shinetsu Silicone Co., Ltd.) which is injected in a microchannel $\left(w_{c h}=10.7 \mu \mathrm{m}, t_{c h}=6.7 \mu \mathrm{m}\right)$ when a meniscus is standing still. The stationary contact angle is nearly equal to $\theta_{Y}=0$ degree. Therefore, the relationships between the largest angle of roughness of the surface $\theta_{\sigma-\max }$ and the dynamic contact angles $\theta_{Y-A}$ and $\theta_{Y-R}$ are given by

$$
\theta_{Y-A}=\theta_{\sigma-\max }
$$

and

$$
\theta_{Y-R}=0 .
$$

Assuming that the driving force given by Eq. (2) is equal to the pressure $P_{\text {hys }}=F_{\text {hys }} /\left(w_{c h} \cdot t_{c h}\right)$ which is given by the drag force (Eq. (8)) normalized by the cross-sectional area of microchannel $\left(w_{c h} \cdot t_{c h}\right)$, threshold electric field $E_{t h-h}$ witch is caused by the contact angle hysteresis is obtained as follow.

$$
E_{t h-h}=\sqrt{\frac{4 \gamma}{\varepsilon_{2}-\varepsilon_{1}} \frac{1-\cos \theta_{\sigma-\max }}{w_{c h}}}
$$

Figure 10 shows the relationship between the $\theta_{\sigma-\max }$ and the threshold electric field $E_{t h-h}$ which is obtained by substituting the parameters $\gamma=24.4[\mathrm{mN} / \mathrm{m}], \epsilon_{r}=2.8$ of the silicone oil (KF56) and the relative dielectric constant of air $\epsilon_{r}=1.0$ into Eq. (13). For example, in the case of the microchannel width of $w_{c h}=8 \mu \mathrm{m}$, the threshold electric field $E_{t h-h}$ of $20[\mathrm{MV} / \mathrm{m}]$ is needed when the angle of side wall

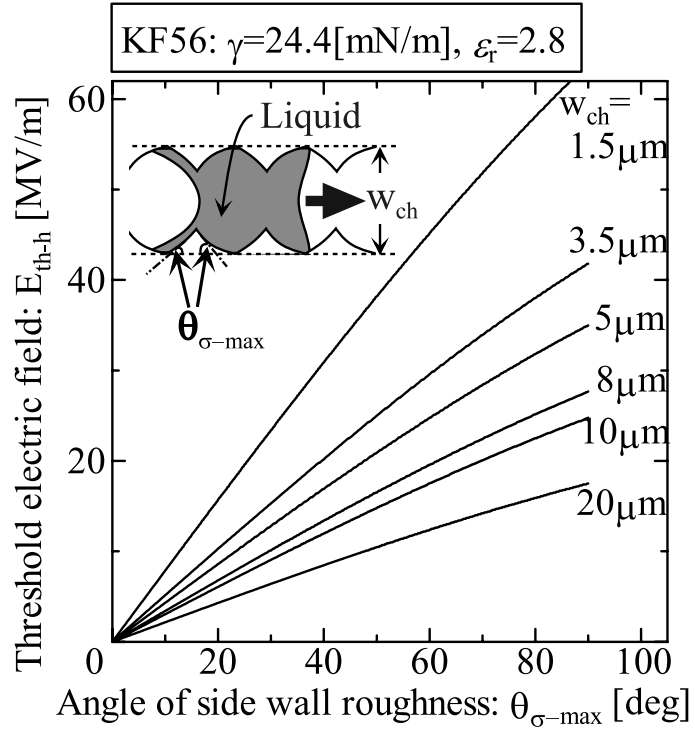

Fig. 10 Calculated result of threshold of electric field caused by contact angle hysteresis.

roughness $\theta_{\sigma-\max }=62$ degrees. The average roughness was evaluated to be 60 degrees, which corresponds to the threshold voltage of about 200 Vwhen the distance of electrodes $d_{\text {elec }}=10 \mu \mathrm{m}$. This voltage is very high for a threshold voltage for practical use, so the side wall roughness must be decreased to reduce threshold voltage.

The disparity of hydraulic diameter between both ends of droplet appears owing to the roughness of the side wall. Resistance force is generated resulting from the capillary phenomenon, when the hydraulic diameter of both ends of droplet is different. It is known as the phenomenon for which a droplet is automatically pulled into a thin pipe. The driving force of droplet must overcome this resistance force, when the droplet is driven by pressure.

The tensional force of interface $F_{\text {capi }}$, that stretches to the subtense of wetted perimeter $2\left(w_{c h}+t_{c h}\right)$, is given in terms of surface tension $\gamma$ by

$$
F_{\text {capi }}=\gamma \cdot[\text { wetted perimeter }]=2 \gamma\left(w_{c h}+t_{c h}\right)
$$

When the widths of microchannel at both ends of droplet are different by $\pm 2 \sigma_{w_{c h}}$ from $w_{c h}$, the difference of pressure between both ends of droplet is given by the following equation.

$$
\Delta P_{\text {capi }}=\frac{4 \gamma \sigma_{w_{c h}}}{w_{c h}\left(w_{c h}-2 \sigma_{w_{c h}}\right)}
$$

Assuming that the driving force given by Eq. (2) is equal to the $\Delta P_{\text {capi }}$ given by Eq. (15), the threshold electric field $E_{t h-c}$ resulting from the capillary phenomenon is obtained as follow.

$$
E_{t h-c}=\sqrt{\frac{8 \gamma \sigma_{w_{c h}}}{\left(\varepsilon_{2}-\varepsilon_{1}\right) w_{c h}\left(w_{c h}-2 \sigma_{w_{c h}}\right)}}
$$

Figure 11 shows the relationship between the $\sigma_{w_{c h}}$ and 


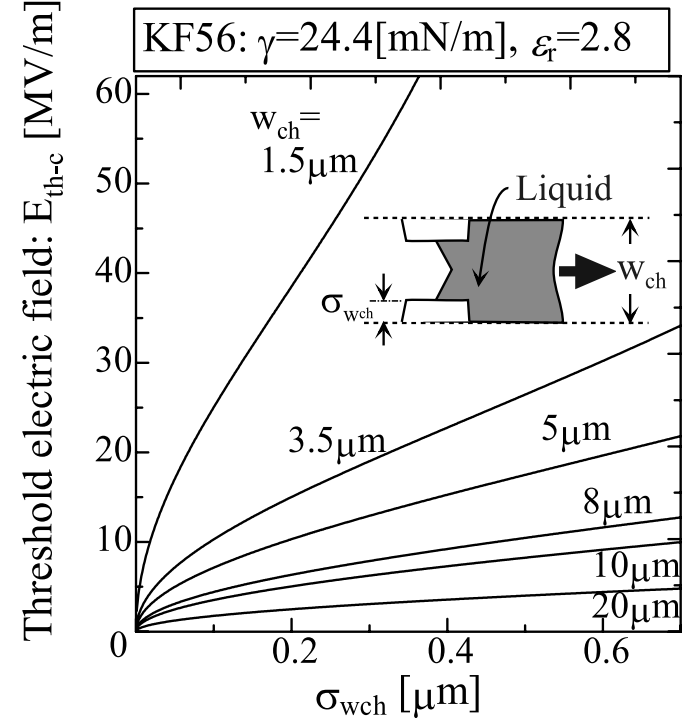

Fig. 11 Calculated result of threshold of electric field caused by capillarity.

the threshold electric field $E_{t h-c}$ which is obtained by substituting the parameters $\gamma=24.4[\mathrm{mN} / \mathrm{m}], \epsilon_{r}=2.8$ of the silicone oil (KF56) and the relative dielectric constant of air $\epsilon_{r}=1.0$ to Eq. (16).

For example, in the case of the microchannel width of $w_{c h}=8 \mu \mathrm{m}$, the threshold electric field $E_{t h-c}$ of $10[\mathrm{MV} / \mathrm{m}]$ is needed when the hight of side wall roughness $\sigma_{w_{c h}}=0.5 \mu \mathrm{m}$. The average of the hight of side wall roughness was evaluated to be $0.5 \mu \mathrm{m}$. This threshold electric field corresponds to $100 \mathrm{~V}$ when the distance of electrodes $d_{\text {elec }}=10 \mu \mathrm{m}$. This voltage is very high threshold voltage for the practical use, too. The roughness height of sidewall $\sigma_{w_{c h}}$ becomes smaller when the angle of side wall becomes smaller. Thus, in order to reduce the threshold voltage, it is necessary to reduce the side wall roughness.

As discussed above, there are two threshold electric fields, i.e., one resulting from the capillary phenomenon and the other is $E_{t h-h}$ resulting from the contact angle hysteresis. The total threshold electric field $E_{t h}$ is given by

$$
E_{t h}=E_{t h-h}+E_{t h-c} .
$$

It is also seen from Figs. 10 and 11 that the threshold electric field can be reduced when the microchannel width is wide. Therefore, in terms of reducing the driving voltage, planular microchannel is suitable as for the continuous fluid model mentioned in the previous paragraph.

\subsection{Droplet Flow Model}

It can be concluded from the above mentioned analysis that the relationship between the flow velocity $U_{0}$ and the driving electric field $E$ is given by

$$
\frac{U_{0} \cdot L_{l q}}{D_{H}^{2}}=\frac{\varepsilon_{2}-\varepsilon_{1}}{64 \mu}\left(E-E_{t h}\right)^{2}
$$

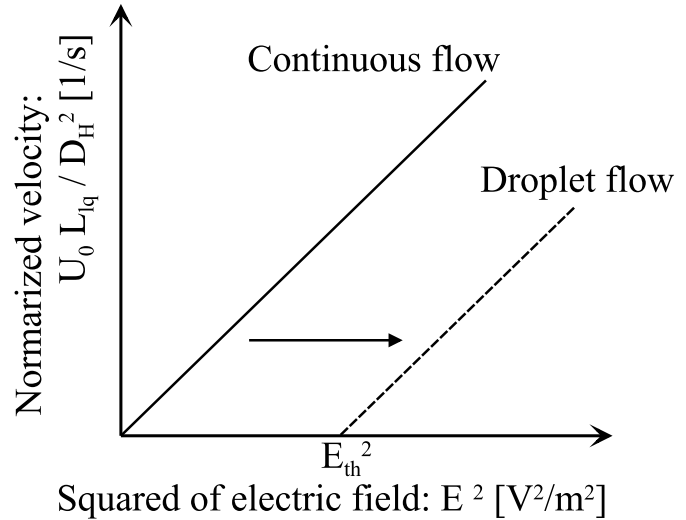

Fig. 12 Comparison of theoretical flow velocity vs applied voltage relation by continuous flow model and droplet flow model.

which is the combination of Eq. (7) for the continuous flow and Eq. (17) which gives the threshold voltage. Here, $D_{H}$ is the hydraulic diameter of the microchannel which is given by Eq. (4). Thus, the relationship between the square of the electric field and the normalized velocity of droplet is given as shown in Fig. 12. In the liquid droplet model a threshold value exists in the electric field, while in the continuous fluid model which does not have the boundary the normalized velocity is proportional to the square of electric field.

A planular microchannel is suitable to obtain a low threshold electric field and a fast velocity. However in a planular microchannel the transmission loss of light beam increases. Therefore in the early stage when the fabrication technology of microchannel is not matured enough, the microchannel should be designed to be planular shape to obtain a low driving voltage suited for practical use. When the fabrication technology is improved and the side wall roughness is reduced enough, then the microchannel should be redesigned to reduce the transmission loss.

\subsection{Relation Between Velocity and Optical Response}

In this section, the relationship between the velocity of liquid droplet $U_{0}$ and the fall time of light transmission $t_{f}$ (or, rise time $t_{r}$ ) is made clear.

For example, when the state of switch is changed from reflection to transmission state, the light output in the crossed port decreases with the movement of matching oil droplet. When the power distribution of waveguide mode in the horizontal direction is designated by $f^{2}(x)$, the transmitted power $F\left(X_{l q f}\right)$ is expressed by

$$
F\left(X_{\mathrm{lqf}}\right)=\frac{\int_{-\infty}^{X_{\mathrm{lqf}}} f^{2}(x) d x}{\int_{-\infty}^{\infty} f^{2}(x) d x}
$$

$X_{l f q}$ is the normalized parameter corresponding to the position of boundary of the droplet measured from the center of waveguide and normalized by the horizontal spot size $w_{0}^{h}$ as shown in the inset of Fig. 13. Figure 13 shows the relationship between $f^{2}(x)$ and $F\left(X_{l q f}\right)$. 


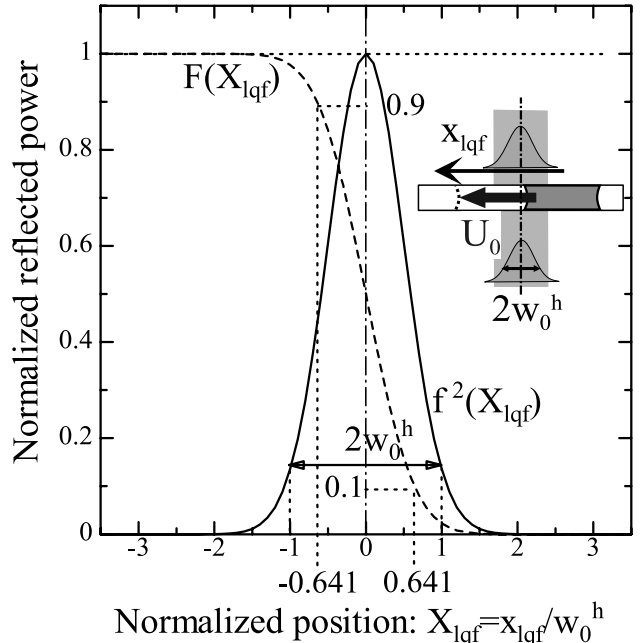

Fig. 13 Relation between modal field profile andtime response signal.

Since the fall time $t_{f}$ is defined as the transition time of waveform change from 90 percent to 10 percent, the fall time $t_{f}$ is expressed in terms of the horizontal spot sizes of the waveguide $w_{0}^{h}$, the drift speed of the droplet $U_{0}$, the crossing angle $\theta_{\text {in }}$ between the microchannel and the waveguide by

$$
t_{f}=\frac{1.282 w_{0}^{h} / \sin \theta_{i n}}{U_{0}} .
$$

Here a similar expression is also obtained for the rising time $t_{r}$.

Therefore, using Eqs. (18) and (20), the following relationship between the electric field $E$ and the fall time $t_{f}$ is obtained:

$$
\frac{t_{f}}{L_{l q}}=82.048 \frac{\mu}{\varepsilon_{2}-\varepsilon_{1}} \frac{w_{0}^{h}}{\sin \theta_{\text {in }}} \frac{1}{D_{H}^{2}} \frac{1}{\left(E-E_{t h}\right)^{2}}
$$

where $L_{l q}$ is the length of the liquid droplet.

\subsection{Distance between Electrodes}

We have shown that the planular microchannel is suited to decrease the driving voltage, since it can shorten the distance between electrodes. However, when the distance between electrodes is shortened too much, the absorption loss due to the metal electrode increases. In this section, the restriction condition of the distance between electrodes is derived.

The modal field profile of the fundamental mode is approximately expressed by the Gaussian profile given by

$$
E(x)=\exp \left[-\left(\frac{x}{w_{0}}\right)^{2}\right]
$$

If the electrode is located at the position of $x=x_{\text {elec }}$, the blade tip part of the modal field further than $x_{\text {elec }}$ is absorbed. The ratio of the blade tip part to the total electric power is

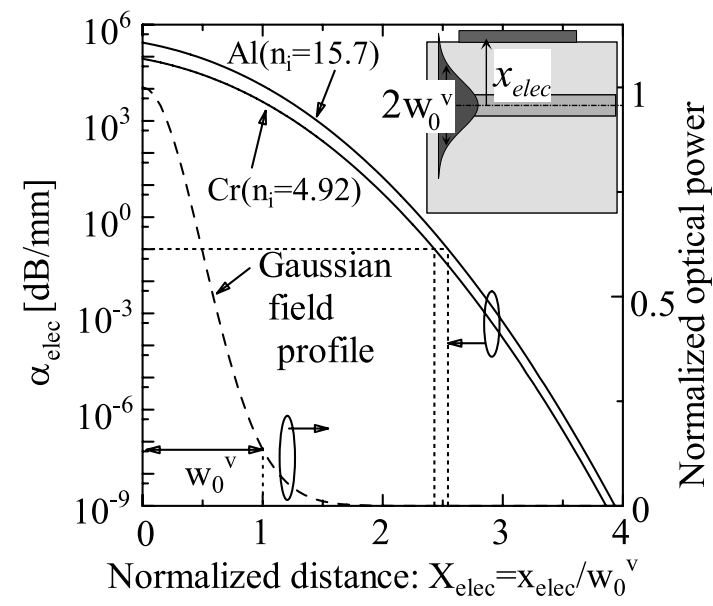

Fig. 14 Absorption loss vs distance between electrodes normarized by spot size.

expressed by

$$
\Gamma_{\text {elec }}\left(x_{\text {elec }}\right)=\frac{\int_{x_{\text {elec }}}^{\infty} E^{2}(x) d x}{\int_{-\infty}^{\infty} E^{2}(x) d x} .
$$

Using the attenuation factor of the electrode material (imaginary part of complex refractive index), the absorption coefficient $\alpha_{\text {elec }}$ can be calculated by the following equation.

$$
\alpha_{\text {elec }}=4.34 \frac{4 \pi n_{i}}{\lambda} \Gamma_{\text {elec }}\left(x_{\text {elec }}\right) \quad[\mathrm{dB} / \mathrm{m}]
$$

where $n_{i}$ is the imaginary part of refractive index of the electrode material $n=n_{r}+j \cdot n_{i}$.

Figure 14 shows the analytical result of the relationship between the absorption loss and the distance of electrodes $X_{\text {elec }}$, which is normalize by the spot size $w_{0}$ as $X_{\text {elec }}=x_{\text {elec }} / w_{0}$. This figure is obtained by substituting the attenuation factors of $\mathrm{Cr}$ and $\mathrm{Al}$, which were used as the electrode materials, into Eq. (24). Here, $n_{A l}=1.42+j 15.7$ and $n_{C r}=4.18+j 4.92$ were used. The electrode length on the top of the waveguide was at longest $100 \mu \mathrm{m}$. It is seen from Fig. 14 that the distance between electrodes should be 2.4 times longer than the spot size $w_{0}^{v}$, in order to make the absorption loss due to the electrode to be less than $0.01 \mathrm{~dB}$, which is negligibly small value.

Figure 14 shows the normalized distance of 2.4 corresponding to the absorption of $0.1[\mathrm{~dB} / \mathrm{mm}]$, which leads to the loss of less than $0.01 \mathrm{~dB}$ because the interaction length with the electrode is less than $100 \mu \mathrm{m}$.

\section{Measurement}

The optical microscope photo of the surface of optical switch and the SEM image of micro channel are shown in Figs. 15 and 16, respectively. The core thickness and width were $0.8 \mu \mathrm{m}$ and $10 \mu \mathrm{m}$, respectively. The microchannel thickness and width were $6.7 \mu \mathrm{m}$ and $8 \mu \mathrm{m}$, respectively.

Figure 17 shows the measured fall waveform in the 


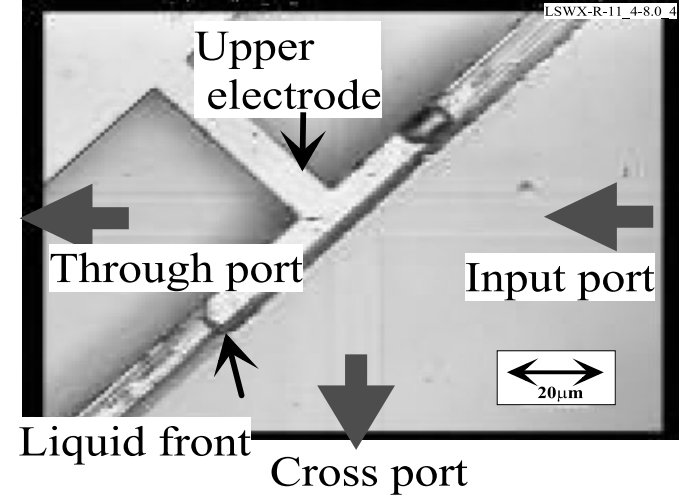

Fig. 15 Top view of optical switch after the injection of matching oil.

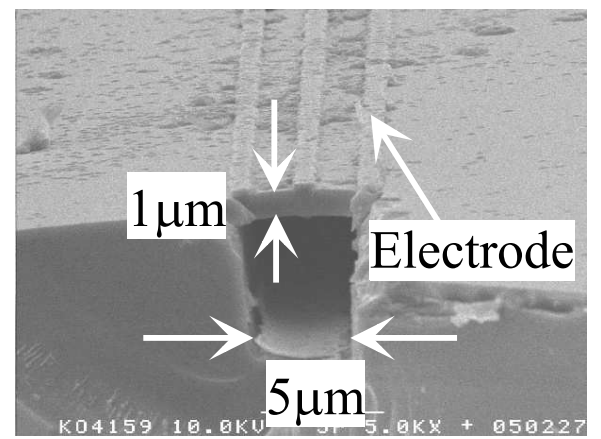

Fig. 16 SEM image of microchannel.

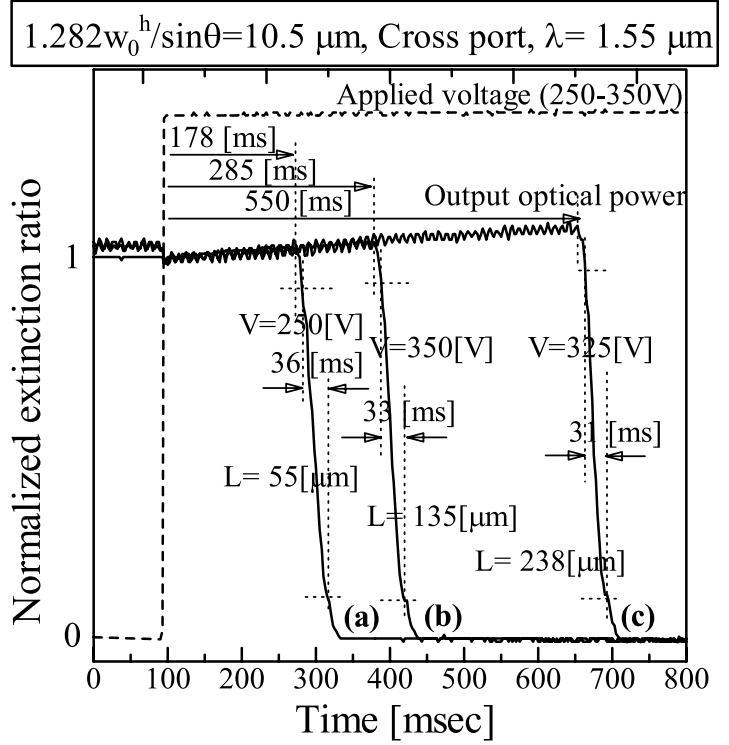

Fig. 17 Measured time response signal of optical switch (cross port, from ON to OFF state).

cross port. The distance from the end face of the liquid droplet to the waveguide intersection part was measured prior to the measurement of the fall response. The measurement was done in the order of (a), (b) and (c) marked on the side of each response waveform shown in Fig. 17. The switching voltage ranged from $200 \mathrm{~V}$ to $350 \mathrm{~V}$.

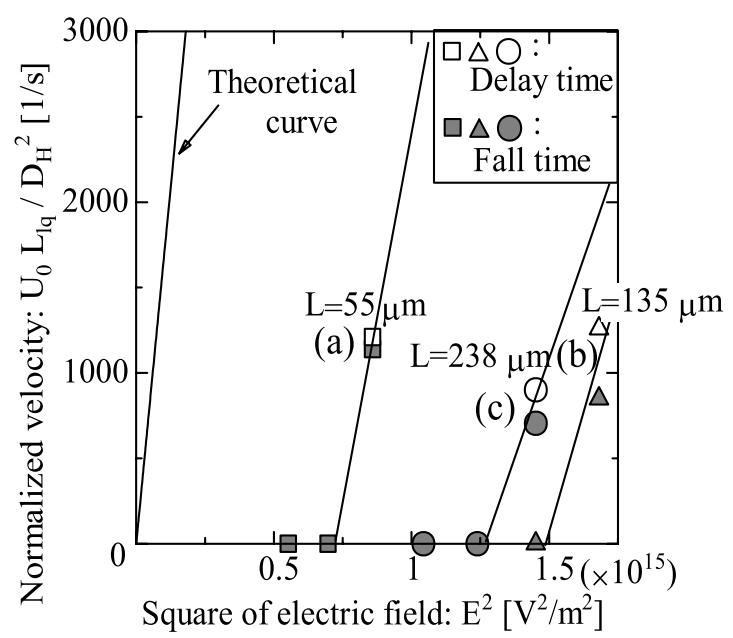

Fig. 18 Normalrized velocity vs square of applied voltage.

In the reflection state in which no liquid droplet is located in the waveguide intersection part, the applied voltage was increased every $25 \mathrm{~V}$ stepwise observing the movement of droplet. If the optical output was not changed, i.e. the liquid droplet was not moved, the applied voltage was increased by $25 \mathrm{~V}$. For the step-like voltage, the delay of fall time was observed corresponding to the starting position of the boundary of droplet. In addition, the fall time of waveform ranged from 31 to $36 \mathrm{~ms}$, which can be regarded almost constant.

The drift speed of liquid droplet $U_{0}^{(\text {delay })}$ is evaluated in terms of the delay time $t_{\text {delay }}$ and the distance $d_{l q}$ from the liquid droplet boundary to the waveguide intersection part by

$$
U_{0}^{(\text {delay })}=\frac{d_{l q}}{t_{\text {delay }}} .
$$

In addition, the verocity of flux $U_{0}^{(f a l l)}$ is evaluated from Eq. (20) using the measured fall time and the horizontal spot size of the waveguide.

The drift speed of liquid droplet which was evaluated from $t_{f}$ in Fig. 17 using Eq. (20) ranged from 0.3 to $0.4 \mathrm{~mm} / \mathrm{s}$, which almost coincided with the value evaluated from the measured $t_{\text {delay }}$ in Fig. 17 using Eq. (25). Figure 18 shows the plots of the evaluated values of drift speed, which is normalized by the liquid droplet length $(135-254 \mu \mathrm{m})$ and the hydraulic diameter of microchannel $D_{H}$, against the square of electric field. It is seen that the threshold electric field was observed as predicted theoretically in Fig. 12 and the slope of the curve is very close to the theoretical value obtained Eq. (18). Therefore, it can be concluded that the experimental result agreed well with the theoretical result mentioned in the previous section.

\subsection{Acceleration of Response Speed}

The measured fall time of 31-36 ms shown in Fig. 17 is not fast enough. This is caused by the large horizontal spot size 


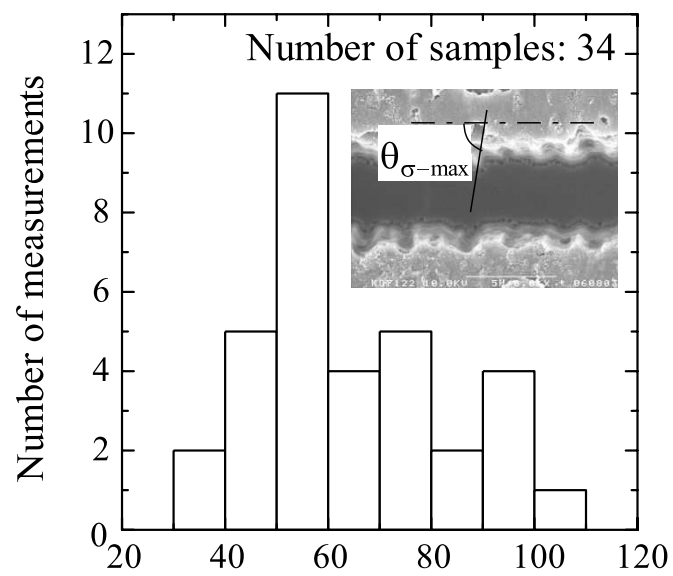

Angle of side wall roughness: $\theta_{\sigma-\max }[\mathrm{deg}]$

Fig. 19 Distribution of largest angle of side wall roughness.

of $4.1 \mu \mathrm{m}$. The acceleration of response speed is possible by decreasing the horizontal spot size $w_{0}^{h}$ as seen from Eq. (21).

The liquid droplet drift speed $U_{0}$ necessary for the switching speed of $10 \mathrm{~ms}$, which is required for the stream type optical switch, is evaluated to be $0.272 \mathrm{~mm} / \mathrm{s}$ from Eq. (20) assuming that the horizontal spot size is $1.5 \mu \mathrm{m}$. The liquid droplet speed evaluated from the measured response time was $0.3-0.4 \mathrm{~mm} / \mathrm{s}$, which satisfies the condition. Therefore, if the horizontal spot size is reduced to $1.5 \mu \mathrm{m}$, the switching time of less than $10 \mathrm{~ms}$ can be expected.

\subsection{Reduction of Threshold Voltage}

The largest angle (tip angle) of the shape of individual side wall roughness was measured using the SEM photograph of fabricated microchannel surface. The SEM image photo of the microchannel is shown in the inset of Fig. 19. The sampled distribution of largest angle of side wall roughness is shown in Fig. 19.

Referring to the curve corresponding to the groove width of $8 \mu \mathrm{m}$ in Fig. 10, the threshold electric field is evaluated to be $10-32 \mathrm{MV} / \mathrm{m}$. When distance between electrodes is $10 \mu \mathrm{m}$, the threshold voltage is evaluated to be $100-320 \mathrm{~V}$.

The distance between electrodes was $8.7 \mu \mathrm{m}$ (air layer $\left(\varepsilon_{r}=1.0\right): 6.7 \mu \mathrm{m}$ and $\left.\mathrm{SiO}_{2}\left(\varepsilon_{r}=3.8\right): 7.7 \mu \mathrm{m}\right)$, the experimental result of threshold voltage agrees well with the theoretical values evaluated from the side wall roughness. To make the driving voltage below $50 \mathrm{~V}$, the threshold voltage must be below $25 \mathrm{~V}(50 \mathrm{~V}-25 \mathrm{~V})$ taking into account the fact that the voltage needed to generate the flow is $25 \mathrm{~V}$. When the distance between electrodes is $7.2 \mu \mathrm{m}$, which can be calculated as $d_{\text {elec }}=4.8 \times 1.5=7.2 \mu \mathrm{m}$ using $d_{\text {elec }}=4.8 \times w_{0}^{v}$ from Fig. 14 and $w_{0}=1.5 \mu \mathrm{m}$, threshold electric field $E_{t h}$ must be below $3.5 \mathrm{MV} / \mathrm{m}$. From the curve corresponding to $8 \mu \mathrm{m}$ in Figs. 10, it is necessary to make the largest angle of side wall roughness $\theta_{\sigma \text {-max }}$ to be less than 10 degrees.

It is seen from Eq. (7) that the voltage needed to generate the same flow flux can be decreased if the liquid droplet length $L_{l q}$ is shortened [12]. When the liquid droplet length $L_{l q}$ is shortened by $1 / 25$ from $250 \mu \mathrm{m}$ to $10 \mu \mathrm{m}$, the electric field needed to generate the same flow flux is reduced by $1 / 5$ and the driving voltage is reduced to $5 \mathrm{~V}$. Thus, the threshold voltage is relaxed to $45 \mathrm{~V}$ and the condition of largest angle of side wall roughness is relaxed to 19 degrees.

To decrease the angle of side wall roughness, it is necessary to improve fabrication conditions of deep groove in the fabrication process of microchannel. It can be realized by the formation of smoother RIE mask and the search of RIE condition suited for deep etching of $\mathrm{SiO}_{2}$.

\section{Conclusion}

The driving condition of the liquid droplet in a microchannel was analyzed from aeromechanics point of view and the design guide-line to obtain satisfactorily good switching property was elucidated.

It was also elucidated that in the movement of liquid droplet the threshold exists in the driving voltage due to the contact angle hysteresis unlike the continuous fluid model (Hagen Poiseuille flow). When the applied voltage is greater than the threshold voltage, the velocity of droplet is proportional to the square of applied voltage as in the case of the continuous flow model.

To decrease the threshold voltage and to increase the drift velocity of droplet, a microchannel with planular cross section is suited. However, since in the planular microchannel the optical loss increases due to the diffraction in the microchannel, there is a trade-off relation between the reduction of optical loss and the reduction of driving voltage. Therefore to decrease the optical loss it is necessary to design the microchannel with high aspect ratio (height greater than width) and the fabrication technology must be improved to reduce the threshold voltage by decreasing the side wall roughness.

We have shown that the threshold voltage exists for the movement of the droplet driven by electric voltage, as predicted by aeromechanics. When the voltage greater than the threshold voltage is applied, the drift velocity of liquid droplet which corresponds to the switching speed agreed well with the theoretical value. The threshold voltage obtained by the experiment was close to the theoretical value evaluated from the angle of side wall roughness of the microchannel.

The optical stream switch with the response speed of less than $10 \mathrm{~ms}$, insertion loss of less than $2 \mathrm{~dB}$, and the driving voltage of less than $50 \mathrm{~V}$ can be realized by the improvement of fabrication process enabling a smooth sidewall of microchannel. This improvement of fabrication process is possible by improved etching condition of deep groove and smooth etching mask.

\section{Acknowledgments}

This work was supported in part by the 21st Century COE Program in the Ministry of Education, Culture, Sports, Sci- 
ence and Technology. The authors also express their sincere thanks to Prof. Masao Washizu of Tokyo Univ. for fruitful discussion.

\section{References}

[1] S. Nagaoka, "Compact latching-type single-mode-fiber switches fabricated by a fiber-micromachining technique and their practical applications," IEEE J. Sell. Top. Quantum Electron., vol.5, no.1, pp.36-45, Jan./Feb., 1999.

[2] J. Kim, C.J. Nuzman, B. Kumar, D.F. Lieuwen, J.S. Kraus, A. Weiss, C.P. Lichtenwalner, A.R. Papazian, R.E. Frahm, N.R. Basavanhally, D.A. Ramsey, V.A. Aksyuk, F. Pardo, M.E. Simon, V. Lifton, H.B. Chan, M. Haueis, A. Gasparyan, H.R. Shea, S. Arney, C.A. Bolle, P.R. Kolodner, R. Ryf, D.T. Neilson, and J.V. Gates, "1100 × 1100 port MEMS-based optical crossconnect with 4-dB maximum loss," IEEE Photonics Technol. Lett., vol.15, no.11, pp.1537-1539, Nov. 2003.

[3] T. Sakata, H. Togo, M. Makihara, F. Shimokawa, and K. Kaneko, "Improvement of switching time in a thermocapillarity optical switch,” J. Lightwave Technol., vol.19, no.7, pp.1023-1027, July 2001.

[4] J.E. Fouquet, "Compact optical cross-connect switch based on total internal reflection in a fluid-containing planar lightwave circuit," Tech. Digest Optic. Commun. Conf., ThS1, 2000.

[5] H.R. Shea, A. Gasparyan, H.B. Chan, S. Arney, R.E. Frahm, D. López, S. Jin, and R.P. McConnell, "Effects of electrical leakage currents on MEMS reliability and performance," IEEE Trans. Device and Materials Reliability, vol.4, no.2, pp.198-207, June 2004.

[6] T. Ikemoto and Y. Kokubun, "Optical waveguide switch based on microfluidics driven by electrostatic force," CLEO-PR 2005, no.CThN2-1, Tokyo, Japan, July 2005.

[7] T. Ikemoto and Y. Kokubun, "Fabrication of microchannel with thin cover layer for an optical waveguide MEMS switch based on microfluidics," IEICE Trans. Electron., vol.E90-C, no.1, pp.78-86, Jan. 2007.

[8] R.B. Bird, W.E. Stewart, and E.N. Lightfoot, Transport Phenomena, John Wiley \& Sons, 2001.

[9] Y. Hanaoka, F. Shimokawa, and Y. Nishida, "Low-loss intersecting grooved waveguides with low $\Delta$ for a self-holding optical matrix switch,” IEEE Trans. Compon. Packag. Manuf. Technol. Part B vol.18, no.2, pp.241-244, May 1995.

[10] C.G. L. Furmidge, "Studies at phase interfaces - I. The sliding of liquid drops on solid surfaces and a theory for spray retention," $\mathrm{J}$. Colloid Science, vol.17, pp.309-324, 1962.

[11] K. Katoh and T. Azuma, "Wetting on a plate with three-dimensional random heterogeneity and roughness: Contact angle hysteresis," Trans. Japan Society of Mechanical Engineers. B, vol.67, no.654, pp.501-507, Feb. 2001.

[12] S.K. Cho, H. Moon, and C.-J. Kim, "Creating, transporting, cuttin and merging liquid droplet by electrowetting-based actuation for digital microfluidic circuits," J. Microelectromechanical Systems, vol.12, no.1, pp.70-80, Feb. 2003.

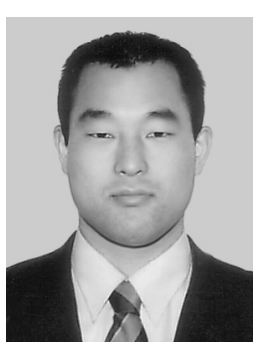

Takuji Ikemoto was born in Tokushima, Japan, on April 15, 1978. He received his B.E. and M.E. degrees in Electrical and Computer Engineering from Yokohama National University, Yokohama, Japan, in 2001 and 2003, respectively. He is working on an optical waveguide switch based on microfluidics towards his Ph.D. degree.

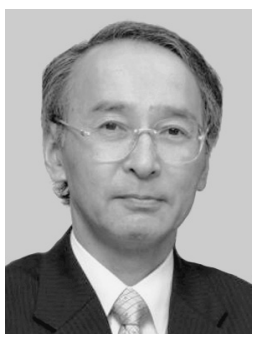

Yasuo Kokubun received his B.E. degree from Yokohama National University, Yokohama, Japan, in 1975 and M.E. and Dr.Eng. degrees from Tokyo Institute of Technology, Tokyo, Japan, in 1977 and 1980, respectively. After he worked for the Research Laboratory of Precision Machinery and Electronics, Tokyo Institute of Technology, as a research associate from 1980 to 1983 , he joined the Yokohama National University as an associate professor in 1983, and is now a professor in the Department of Electrical and Computer Engineering. From 2006 he is serving as the dean of the Faculty of Engineering. His current research is in integrated photonics, particularly waveguide-type functional devices and threedimensional integrated photonics. From 1984 to 1985 he was with AT\&T Bell Laboratories, Holmdel, NJ, as a visiting reseacher and was engaged in the study of a novel waveguide on a semiconductor substrate (ARROW) for integrated optics. From 1996 to 1999, he served as the project leader of the "Three-dimensional microphotonics" project at the Kanagawa Academy of Science and Technology." Professor Kokubun is a senior member of the Institute of Electrical and Electronics Engineers, a member of the Japan Society of Applied Physics, and the Optical Society of America. 